\title{
Local Nasal Specific Immunotherapy for Allergic Rhinitis
}

\author{
Giovanni Passalacqua, MD; Giorgio Walter Canonica, MD
}

\begin{abstract}
The possibility of producing local hyposensitization by administering allergens via mucosal routes was envisaged at the beginning of 1900, and local nasal immunotherapy has been extensively studied since the 1970s. Presently, there are 21 randomized controlled trials being conducted with the most common allergens, consistently showing the clinical efficacy of local nasal immunotherapy for rhinitis. Other advantages are that it has an optimal safety profile and can be self-administered at home by the patient. Moreover, there are several data from animal models and from humans that confirm the immunomodulatory effect of intranasally administered antigens. On the other hand, local nasal immunotherapy seems to be effective only on rhinitis symptoms and requires a particular technique of administration. For these reasons, its clinical use is progressively declining in favour of the sublingual route although nasal immunotherapy is validated in official documents and remains a viable alternative to injection.
\end{abstract}

\section{Historical Background}

Since the first empirical attempts at the beginning of the twentieth century, ${ }^{1}$ specific immunotherapy (SIT) for allergic rhinitis has been administered via the subcutaneous route. The clinical efficacy of this treatment was immediately recognized, and its use rapidly spread. At the same time, attempts were made to administer the allergenic extracts via different routes, and in fact, oral immunotherapy was first proposed in the early 1900 s. $^{2,3}$ Later, during the 1950 s, local bronchial desensitization was suggested and investigated $^{4,5}$ whereas sublingual immunotherapy

G. Passalacqua and G.W. Canonica-Allergy and Respiratory Diseases, Dept. of Internal Medicine, University of Genoa, Genoa, Italy;

Correspondence to: Giovanni Passalacqua, MD, Allergy and Respiratory Diseases, Dept. of Internal Medicine, University of Genoa, Padiglione Maragliano, L.go R. Benzi 10, 16132 Genoa, Italy

DOI 10.2310/7480.2006.00010
(SLIT) appeared only in $1986 .{ }^{6}$ The main stimulus to the development of noninjection routes was the problem of the safety of injected SIT, which became of primary relevance after the formal and detailed report of numerous deaths due to subcutaneous SIT. ${ }^{7}$ Nevertheless, independent of the safety issue, local nasal immunotherapy (LNIT) has been extensively investigated since the $1970 \mathrm{~s},{ }^{8,9}$ first in the United States and later in Italy. Within the last 20 years, reports from more than 60 controlled trials of various noninjection routes have been published in peer-reviewed journals, but in 1998, on the basis of an extensive review of the literature, a panel of experts from the World Health Organization concluded that only SLIT and LNIT are viable alternatives to therapy by the injection route. ${ }^{10}$ These conclusions were soon confirmed in a position paper by the European Academy of Allergology and Clinical Immunology ${ }^{11}$ and in the ARIA (Allergic Rhinitis and Its Impact on Asthma) study document. ${ }^{12}$ Currently, SLIT is prescribed in many European countries whereas the use of LNIT is progressively decreasing, mainly because of technical limitations. 


\begin{tabular}{|c|c|c|c|}
\hline Wk 1-2 & Wk 3-4 & Wk 5-6 & Wk 7-8 \\
\hline Vial 1 & Vial 2 & Vial 3 & Vial 4 \\
\hline $125 \mathrm{UB} / \mathrm{mL}$ & $250 \mathrm{UB} / \mathrm{mL}$ & $500 \mathrm{UB} / \mathrm{ml}$ & $1.000 \mathrm{UB} / \mathrm{mL}$ \\
\hline DAY & DAY & DAY & DAY \\
\hline $1 \triangleq$ & $1 \Leftarrow$ & $1 乞$ & $1 \stackrel{\bullet}{\bullet}$ \\
\hline 2 & 2 & 2 & 2 \\
\hline $3 \Leftarrow$ & $3 \Leftarrow$ & $3 \curvearrowleft$ & $3 \smile$ \\
\hline 4 & 4 & 4 & 4 \\
\hline $5 \Leftarrow$ & $5 \Leftarrow$ & $5 \curvearrowleft$ & $5 \smile$ \\
\hline 6 & 6 & 6 & 6 \\
\hline 7 & 7 & 7 & 7 \\
\hline
\end{tabular}

Maintenance:

1 puff in one nostril from vial 4 on alternate days

Figure 1 Example of an administration schedule for local nasal immunotherapy (LNIT) with an aqueous extract prepared at different concentrations. $\mathrm{UB}=$ biologic units; $\mathrm{Wk}=$ weeks.

\section{Practical Aspects}

LNIT is the administration (by spray) of gradually increasing amounts of allergen (build-up or up-dosing phase) into the nasal cavity until a maintenance dose is reached. The maintenance dose is then given continually at variable intervals to maintain "hyposensitization" and to reduce symptoms due to natural exposure to the allergen. The few nasal vaccines are commercialized in Europe and standardized either biologically or immunologically. Like the extracts used for subcutaneous immunotherapy, they are labelled in arbitrary units, according to in-house references. LNIT is given as a nasal spray that can be either an aqueous solution or a dry powder. LNIT can be administered either preseasonally (stopping at the beginning of the season), "pre-coseasonally" (stopping at the end of the season), or continually. Pre-coseasonal schedules are the choice for pollen allergy whereas continuous treatments are preferred for perennial allergens. The build-up phase usually lasts about 4 to 6 weeks, and the vaccine is prepared in separate vials or blisters at increasing concentrations (Figure 1). Simplified schedules of administration (steady dosage) have also been proposed, to make LNIT more "patient friendly." Detailed education of the patient is mandatory because LNIT is self-administered at home by the patient. Administration requires good train-
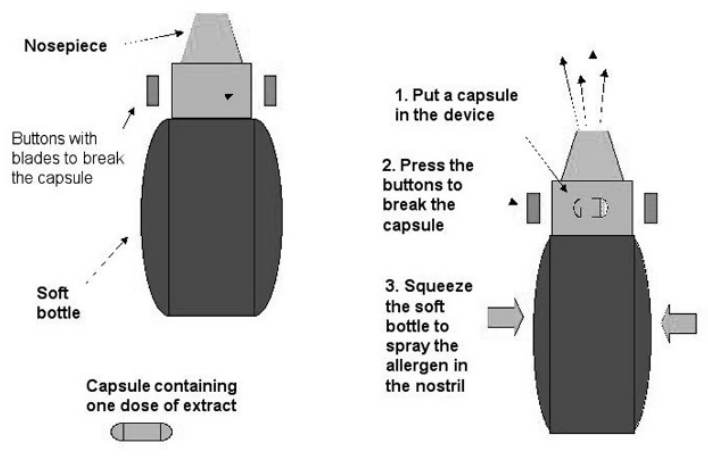

Figure 2 Diagram of a delivery device for extracts prepared as dry-powder capsules (Allerkin, Lofarma S.p.A, Milan, Italy).

ing and some precautions so that inhalation into the deep airways (ie, the patient's vocalizing while spraying) is avoided. Dry-powder capsules used with a spraying device make administration quite easy (Figure 2). Some authors have suggested premedication with nasal cromolyn before each dose, but there is no strict scientific support for this.

\section{Efficacy and Safety}

To date, there have been 21 randomized doubleblind placebo-controlled studies with LNIT (Table 1). ${ }^{13-33}$ All but two ${ }^{16,24}$ invariantly documented a significant reduction of symptoms and/or drug intake scores. In this regard, the clinical efficacy of LNIT for rhinitis symptoms was comparable to that of subcutaneous SIT. In parallel, several trials demonstrated a measurable reduction of the nasal response to specific challenge, ${ }^{24-32}$ confirming the effectiveness of hyposensitization. The large majority of LNIT studies were conducted among subjects with pollinosis, and the treatment was proved to be effective with all the major pollens (including birch, ragweed, grasses, and Parietaria judaica). There were also four studies performed with a mite extract, three with favourable results ${ }^{20,31,32}$ and one with negative results, ${ }^{24}$ but in this latter study, LNIT was admin- 
Table 1 Double-Blind Placebo-Controlled Studies of Local Nasal Immunotherapy

\begin{tabular}{|c|c|c|c|c|c|}
\hline Author, Year & Age Range (yrs) & $\begin{array}{c}\text { No. of } \\
\text { Patients } \\
\text { (Active/ } \\
\text { Placebo) }\end{array}$ & Allergen & Duration & Type of Extract \\
\hline Johansson, $1979^{13}$ & $\mathrm{Ad}$ & $12 / 11$ & Grass & $14 \mathrm{wk}$ & Aqueous, modified \\
\hline Nickelsen, $1981^{14}$ & $16-66$ & $38 / 34$ & Ragweed & $3 \mathrm{mo}$ & Aqueous, modified \\
\hline Welsh, $1981^{15}$ & $13-58$ & $18 / 15$ & Ragweed & $20 \mathrm{wk}$ & Aqueous \\
\hline Schumacher, $1982^{16}$ & $20-53$ & 8/7 & Grass & $10 \mathrm{wk}$ & Powder, modified \\
\hline Georgitis, $1983^{17}$ & $16-67$ & $31 / 13$ & Grass & $10 \mathrm{wk}$ & Aqueous, modified \\
\hline Georgitis, $1984^{18}$ & Ad & $29 / 16$ & Grass & $10 \mathrm{wk}$ & Aqueous, modified \\
\hline Andri, $1992^{19}$ & $14-54$ & $8 / 8$ & Parietaria & $18 \mathrm{wk}$ & Powder, modified \\
\hline Andri, $1993^{20}$ & $15-54$ & $11 / 10$ & Mite & $12 \mathrm{mo}$ & Powder \\
\hline Passalacqua, $1995^{21}$ & $20-56$ & $9 / 9$ & Parietaria & $5 \mathrm{mo}$ & Powder \\
\hline D’Amato, $1995^{22}$ & $13-37$ & $10 / 10$ & Parietaria & $8 \mathrm{mo}$ & Powder \\
\hline Andri, $1995^{23}$ & $17-56$ & $14 / 14$ & Birch & $22 \mathrm{wk}$ & Powder \\
\hline Fanales-Belasio, $1995^{24}$ & $16-49$ & $10 / 10$ & Mite & $3 \mathrm{mo}$ & Aqueous \\
\hline Andri, $1996^{25}$ & $14-52$ & $13 / 15$ & Grass & $4 \mathrm{mo}$ & Powder \\
\hline Cirla, $19962^{6}$ & $17-44$ & $11 / 11$ & Birch/alder & 4 mo & Powder \\
\hline Bardare, $1996^{27}$ & $5-15$ & $19 / 20$ & Grass & $3 \mathrm{mo}$ & Powder \\
\hline Gaglani, $1997^{28}$ & $18-35$ & $13 / 14$ & Weed mix & $4 \mathrm{mo}$ & Aqueous \\
\hline Bertoni, $1999^{29}$ & $18-43$ & $10 / 10$ & Grass & $3 \mathrm{mo}$ & Aqueous \\
\hline Motta, $2000^{30}$ & $13-55$ & $55 / 47$ & Grass/mite & $8 \mathrm{mo}$ & Aqueous \\
\hline Pocobelli, $2001^{31}$ & $16-45$ & $22 / 21$ & Grass & $4 \mathrm{mo}$ & Powder, modified \\
\hline Marcucci, $2002^{32}$ & $4-15$ & $16 / 16$ & Mite & $18 \mathrm{mo}$ & Powder, modified \\
\hline Passali, $2002^{33}$ & $16-47$ & $18 / 18$ & Mite & $8 \mathrm{mo}$ & Powder, modified \\
\hline
\end{tabular}

Ad = advanced age; $w k=$ weeks.

istered for 3 months only. Few studies (all with positive results) were carried out with children, ${ }^{27,32}$ and there is insufficient experience with the pediatric age group. LNIT seems to be effective only locally (ie, for rhinitis symptoms); there is no proof that it is also able to treat asthma. There is one study (uncontrolled, with 43 pollinosis patients) that shows a reduction of nonspecific bronchial hyperresponsiveness following LNIT. $^{34}$ Concerning the duration of clinical efficacy, the only available follow-up of a randomized controlled trial suggested that LNIT does not maintain its clinical efficacy once it is discontinued and that a preseasonal course is needed every year. ${ }^{35}$ On the other hand, a prospective observational study (not randomized and not controlled) documented 3 to 5 years of long-lasting clinical efficacy in 22 patients after discontinuation of LNIT. ${ }^{36}$ Aqueous extracts of unmodified allergens are highly effective, but they also provoke mild LNIT- induced rhinitis whereas treatment with allergoids (chemically modified allergens) is almost devoid of local side effects (however, allergoids are less potent). These facts, observed in the earliest studies, raised some concerns about the clinical use of LNIT $^{37}$ because although the symptoms were reduced during the allergen exposure, they were present (even if mild) at every LNIT administration. Extracts that are prepared as dry powder solved the problem; the granules (with a diameter of 40-50 $\mu \mathrm{m}$ ) allow a uniform deposition on the nasal mucosa and do not provoke clinical symptoms. In fact, no side effects or only negligible side effects were reported in all the studies that used dry powders. In one study, three patients withdrew because of bronchospasm after administration, but this side effect was attributed to incorrect technique (ie, improper inhalation of the allergen). ${ }^{22}$ LNIT seems to be well tolerated and to have a reasonable safety profile. ${ }^{11,12}$ 
In the more recent studies, a simplified schedule of administration was used, with a single and steady dosage from the beginning. ${ }^{31,33}$ This administration schedule is advantageous for patients because different preparations at different concentrations are no longer required and because accidental dosing mistakes are avoided. The clinical equivalence between the steady dosage and the traditional build-up was demonstrated in a randomized open trial. ${ }^{38}$

\section{Immunologic Aspects}

As mentioned above, the first attempts to achieve a selective hyposensitization of the nasal mucosa were made at the beginning of the $1970 \mathrm{~s} .{ }^{8} 9$ The underlying rationale was derived from the observation that hyporesponsiveness of the nasal mucosa could be achieved after repeated stimulation with low doses of allergen. ${ }^{39}$ From a clinical viewpoint, this is the opposite of the well-known priming effect, whereby an increased mucosal response is seen after a single administration of allergen. ${ }^{40}$

The changes in nasal allergic response over time were confirmed in a challenge-rechallenge study. ${ }^{41}$ In this trial, allergic patients received a baseline nasal challenge and a rechallenge after 3 , 7,14 , or 28 days. If the rechallenge was made after 3 days, the clinical response was greater than baseline (priming effect) whereas there was a significantly decreased response at 14 days. The 7- and 28-day challenges evoked a clinical response identical to that of the baseline challenge (Figure 3). In general, the local administration of allergens is supported by a number of experimental observations in animal models, showing the "tolerogenicity" of mucosal antigen delivery. ${ }^{42,43}$ In an animal model, it was seen that mucosal administration of the antigen could select a functionally disabled population of $\mathrm{CD}^{+}{ }^{+}$cells. ${ }^{44}$ Also, in animal models, intranasal administration of antigens was found to induce an increased production of interleukin-10, ${ }^{45,46}$ the regulatory and antiinflammatory cytokine involved in the mechanisms of action of traditional SCIT. ${ }^{47,48}$ Systemic immunologic changes induced by LNIT in humans were reported only sporadically in the above-

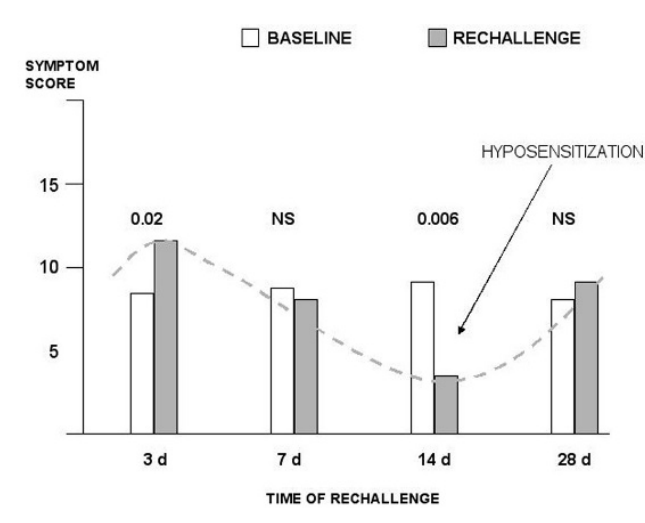

Figure 3 Graph showing how the intensity of nasal response to rechallenge is different at different times. If the rechallenge is made 2 weeks after the first challenge, a significant reduction in the response is seen. Adapted from Nickelsen JA et al. ${ }^{41} \mathrm{~d}=$ days; NS =

mentioned clinical trials. In one open study of LNIT and SCIT, it was shown that only SCIT could induce an increase in circulating immunoglobulin G4. ${ }^{49}$ Nevertheless, patients treated with LNIT also showed decreased proliferation of allergen-specific T-lymphocyte clones after treatment. In one clinical trial, it was shown that LNIT is capable of modulating allergic inflammation by down-regulating the expression of intercellular adhesion molecule $1 .{ }^{35}$ Despite their wide clinical use, nothing is known about the absorption and fate of SCIT allergenic extracts in humans. Indeed, their biodistribution would be of particular importance in the case of local administration. In rats and rabbits, significant absorption of the allergen through sublingual and nasal mucosae has been shown. ${ }^{50}$ This is in agreement with the increased permeability of nasal epithelium to macromolecules that is observed in allergic subjects, ${ }^{51}$ but there is still controversy concerning the pharmacokinetics of allergens. ${ }^{52}$ In recent years, the biodistribution of mucosal antigens was assessed in humans with the use of a radiolabelled purified allergen (Par j 1) and a special procedure. ${ }^{53}$ With this design, it was observed that no direct absorption of the allergen through the sublingual mucosa occurs and that plasma radioactivity increases only after the allergen is swallowed. This is also true for nasal administration. 


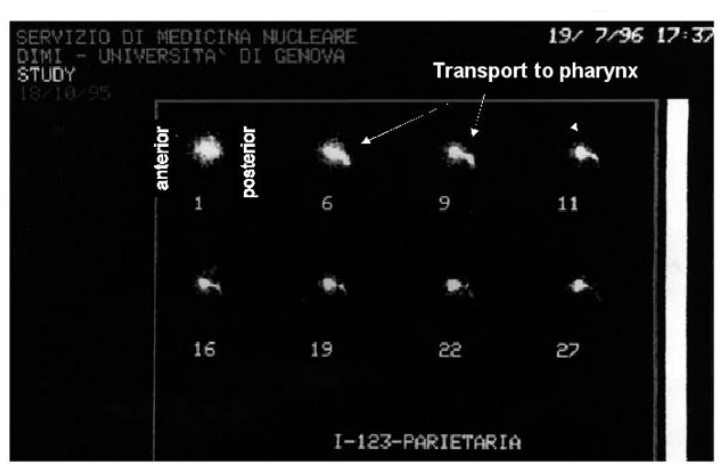

Figure 4 Pharmacokinetics of radiolabelled Par $\mathrm{j} 1$ in a nonallergic volunteer. Sequential scintiscans of the nasal region, recorded at different times (up to $30 \mathrm{~min}-$ utes) after administration, show a progression of the allergen toward the pharynx.

In healthy volunteers, the allergen sprayed into the nose is slowly transported by mucociliary clearance towards the pharynx (Figure 4) and is progressively swallowed, but a fraction is retained for long periods (up to 40 hours) in the mucosa..$^{53,54}$ Things are partly different in allergic subjects; the allergen is cleared from the nose faster than in nonallergic persons, and it disappears completely from the nose within minutes. ${ }^{55}$

\section{Conclusions}

On the basis of the literature review, it can be said that local nasal immunotherapy (LNIT) is clearly effective in treating allergic rhinitis because it alleviates symptoms and reduces the need for concomitant medications during natural exposure to allergens. Another favourable aspect of LNIT is its safety, which has been repeatedly confirmed in numerous trials. Moreover, LNIT is self-administered by patients in their homes, thus avoiding the costs for injections and loss of time. On the other hand, LNIT is effective only for rhinitis whereas patients often have concomitant allergic diseases such as asthma and conjunctivitis. In addition, a specific administration technique is needed to avoid inhalation of the extract into the deep airways whereas sublingual immunotherapy (SLIT) is more easily used and has a systemic effect. For these reasons, the clinical use of LNIT is progressively declining in favour of SLIT. In Italy, LNIT currently accounts for about $10 \%$ of immunotherapy sales. Nevertheless, it remains a viable alternative to subcutaneous therapy. The optimal candidates are adult and well-trained patients with pollen-induced rhinitis and patients who refuse injections or who cannot tolerate the subcutaneous regimen.

\section{References}

1. Noon L. Prophylactic inoculation against hay fever. Lancet 1911;i:1572-3.

2. Black JH. The oral administration of pollen. J Lab Clin Med 1927;12:1156.

3. Black JH. The oral administration of pollen: clinical report. J Lab Clin Med 1928;13:709.

4. Herxeimer H. Bronchial hypersensitization and hyposensitization in man. Int Arch Allergy Appl Immunol 1951;40:40-57.

5. Herxeimer H, Prior EN. Further observations in induced asthma and bronchial hyposensitization. Int Arch Allergy Appl Immunol 1952;3:159-61.

6. Scadding K, Brostoff J. Low dose sublingual therapy in patients with allergic rhinitis due to dust mite. Clin Allergy 1986;16:483-91.

7. Committee on the Safety of Medicines. CSM update. Desensitizing vaccines. Br Med J 1986;293:948.

8. Metha SB, Smith JM. Nasal hyposensitization and hayfever. Clin Allergy 1975;5:279-84.

9. Taylor G, Shivalkar PR. Local nasal desensitization in allergic rhinitis. Clin Allergy 1972;2:125-6.

10. Bousquet J, Lockey R, Malling HJ. Allergen immunotherapy: therapeutic vaccines for allergic diseases. World Health Organization position paper. Allergy 1998;53 Suppl:

11. Malling HJ, Abreu-Nogueira J, Alvarez-Cuesta E, et al. EAACI position paper on local immunotherapy. Allergy 1998;53:933-44.

12. Bousquet J, Van Cauwenberge P. Allergic rhinitis and its impact on asthma. J Allergy Clin Immunol 2001;108:S83-5.

13. Johansson SG, Deuschl H, Zetterstrom O. Use of glutaraldehyde-modified timothy grass pollen extract in nasal hyposensitization of hay fever. Int Arch Allergy Appl Immunol 1979;60:447-60.

14. Nickelsen JA, Goldstein S, Mueller U, et al. Local intranasal immunotherapy for ragweed allergic rhinitis: clinical response. J Allergy Clin Immunol 1981;68:33-40. 
15. Welsh PW, Zimmermann EM, Yunginger JW, et al. Preseasonal intranasal immunotherapy with nebulized short ragweed extract. J Allergy Clin Immunol 1981;67:237-42.

16. Schumacher MJ, Pain MC. Intranasal immunotherapy and polymerized grass pollen allergens. Allergy 1982;37:241-8.

17. Georgitis JW, Reisman RE, Clayton WF, et al. Local nasal immunotherapy for grass allergic rhinitis. J Allergy Clin Immunol 1983;71:71-6.

18. Georgitis JW, Clayton WF, Wypich JI, et al. Further evaluation of local nasal immunotherapy with aqueous and allergoid grass extract. J Allergy Clin Immunol 1984;74:694-700.

19. Andri L, Senna GE, Betteli C, et al. Local nasal immunotherapy in allergic rhinitis to Parietaria. Allergy 1992;47:318-23.

20. Andri L, Senna GE, Betteli C, et al. Local nasal immunotherapy for Dermatophagoides-induced rhinitis: efficacy of a powder extract. J Allergy Clin Immunology 1993;91:587-96.

21. Passalacqua G, Albano M, Ruffoni S, et al. Local nasal immunotherapy to Parietaria: evidence of reduction of allergic inflammation. Am J Respir Crit Care Med 1995;152:461-6.

22. D'Amato G, Lobefalo G, Liccardi G, Cazzola M. A double blind placebo controlled trial of local nasal immunotherapy in allergic rhinitis to Parietaria pollen. Clin Exp Allergy 1995;25:141-8.

23. Andri L, Senna GE, Andri G, et al. Local nasal immunotherapy for birch allergic rhinitis with extract in powder form. Clin Exp Allergy 1995;25:1092-9.

24. Fanales-Belasio E, Ciofalo A, Zambetti G, et al. Intranasal immunotherapy with Dermatophagoides extract: in vivo and in vitro results of a double-blind placebo-controlled trial. Rhinology 1995;33:126-31.

25. Andri L, Senna GE, Betteli C, et al. Local nasal immunotherapy with extract in powder form is effective and safe in grass pollen rhinitis. A double blind study on 32 patients. J Allergy Clin Immunol 1996;97:37-41.

26. Cirla A, Sforza N, Roffi GP, et al. Preseasonal intranasal immunotherapy in birch-alder rhinitis. A double blind study. Allergy 1996;51:299-305.

27. Bardare M, Zaini C, Novembre E, Vierucci A. Local nasal immunotherapy with a powder extract for grass pollen rhinitis in pediatric ages. A controlled study. J Invest Allergol Clin Immunol 1996;97:34-41.
28. Gaglani B, Borish L, Bartelson BL, et al. Nasal immunotherapy in weed-induced allergic rhinitis. Ann Allergy Asthma Immunol 1997;79:259-65.

29. Bertoni M, Cosmi F, Bianchi I, Di Berardino L. Clinical efficacy and tolerability of a steady dosage schedule of local nasal immunotherapy. Results of preseasonal treatment in grass pollen rhinitis. Ann Allergy Asthma Immunol 1999;82:47-51.

30. Motta G, Passali D, De Vincentiis I, et al. A multicenter trial of specific local nasal immunotherapy. Laryngoscope 2000;110:132-9.

31. Pocobelli D, Del Bono A, Venuti L, et al. Nasal immunotherapy at constant dosage: a double blind placebo controlled study in grass allergic rhinoconjunctivitis. J Invest Allergol Clin Immunol 2001;11:79-88.

32. Marcucci F, Sensi LG, Caffarelli C, et al. Lowdose local nasal immunotherapy in children with perennial allergic rhinitis due to Dermatophagoides. Allergy 2002;57:23-8.

33. Passali D, Bellussi L, Passali GC, Passali FM. Nasal immunotherapy is effective in the treatment of rhinitis due to mite allergy. A double-blind, placebo-controlled study with rhinological evaluation. Int J Immunopathol Pharmacol 2002;15:141-7.

34. Olivieri M, Mohaddes Zadeh MR, Talamini G, et al. Local nasal immunotherapy and bronchial hyperreactivity in seasonal allergic rhinitis: an observational pilot study. J Investig Allergol Clin Immunol 2000;10:300-4.

35. Passalacqua G, Albano M, Pronzato C, et al. Nasal immunotherapy to Parietaria: long term follow up of a double blind study. Clin Exp Allergy 1997;27:904-8.

36. Andri L, Falagiani P. Symptomatic relief after grass nasal immunotherapy: lasting efficacy after 4-5 years. J Investig Allergol Clin Immunol 2003;13:228-31.

37. Bjorksten B. Local immunotherapy is not documented for clinical use. Allergy 1994;49:299-301.

38. Senna GE, Andri L, Dama A, et al. Local nasal immunotherapy: efficacy and tolerability of two different administration schedules in grass pollen rhinitis. Allergol Immunopathol (Madr) 2000;28:238-42.

39. Taylor G, Shivalkar E. Changes in nasal airways resistance on antigenic challenge in allergic rhinitis. Clin Allergy 1971;1:63-73.

40. Koh YY, Lim HS, Min KU, Min YG. Airways of allergic rhinitics are 'primed' to repeated aller- 
gen inhalation challenge. Clin Exp Allergy 1994;24:337-46.

41. Ciprandi G, Ricca V, Landi M, et al. Allergen specific nasal challenge: response kinetic of clinical and inflammatory events to rechallenge. Int Arch Allergy Immunol 1998;115:157-61.

42. Holt PG, Sedgwick JD. Suppression of IgE response following antigen inhalation: a natural homeostatic mechanism which limits sensitizations to aeroallergens. Immunol Today 1987;8:14-5.

43. Holt PG, Reid M, Britten D, et al. Suppression of IgE responses by passive antigen inhalation: dissociation of local and systemic immunity. Cell Immunol 1987;104:434-9.

44. Tsitoure DC, DeKruyff RH, Lamb JR, Umetsu DT. Intranasal exposure to antigen induces immunological tolerance mediated by functionally disabled CD4+ T cells. J Immunol 1999;163:2592-600.

45. Barbey C, Donatelli-Dufour N, Batard P, et al. Intranasal treatment with ovalbumin but not the major T cell epitope ovalbumin 323-339 generates interleukin-10 secreting $\mathrm{T}$ cells and results in the induction of allergen systemic tolerance. Clin Exp Allergy 2004;34:654-62.

46. Hall G, Houghton CG. Suppression of allergen reactive $\mathrm{Th} 2$ mediated responses and pulmonary eosinophilia by intranasal administration of an immunodominant peptide is linked to IL-10 production. Vaccine 2003;21:549-61.

47. Till SJ, Francis JN, Nouri-Aria K, Durham SR. Mechanisms of immunotherapy. J Allergy Clin Immunol 2004;113:1025-34.
48. Robinson DS, Larche M, Durham SR. Tregs and allergic disease. J Clin Invest 2004;114:1389-97.

49. Giannarini L, Maggi E. Decrease of allergen specific $T$ cell response induced by nasal immunotherapy. Clin Exp Allergy 1998;28:404-12.

50. Mistrello G, Rapisarda G, Falagiani P. Detection of IgE-binding activity in serum after intranasal treatment of normal rabbits with $P$. judaica extracts. Allergy 1991;46:52-5.

51. Buckle FB, Cohen AB. Nasal mucosal hyperpermeability to macromolecules in atopic rhinitis and extrinsic asthma. J Allergy Clin Immunol 1975;55:213-21.

52. Falagiani P, Mistrello G. Pharmacokinetics of allergens after local administration. Allergy 1997;52:17-21.

53. Bagnasco M, Mariani G, Passalacqua G, et al. Absorption and distribution kinetics of the major Parietaria allergen administered by noninjectable routes to healthy human beings. J Allergy Clin Immunol 1997; 100:122-9.

54. Bagnasco M, Passalacqua G, Villa G. Pharmacokinetics of an allergen and a monomeric allergoid for oromucosal immunotherapy in allergic volunteers. Clin Exp Allergy 2001;31:54-60.

55. Passalacqua G, Altrinetti V, Mariani G, et al. Pharmacokinetics of radiolabelled Par j 1 administered intranasally to allergic and healthy subjects. Clin Exp Allergy 2005;35:880-3. 